\title{
Efficacy and safety of photodynamic therapy versus Nd-YAG laser resection in NSCLC with airway obstruction
}

\author{
J.P. Diaz-Jiménez, J.E. Martínez-Ballarín, A. Llunell, E. Farrero, A. Rodríguez, M.J. Castro
}

\begin{abstract}
Effectiveness and safety of photodynamic therapy versus Nd-YAG laser resection in NSCLC with airway obstruction. J.P. Diaz-Jiménez, J.E. Martnez-Ballarn, A. Llunell, E. Farrero, A. Rodrǵuez, M.J. Castro. (C) ERS Journals Ltd 1999.

ABSTRACT: A prospective controlled randomized trial was performed in order to assess the effectiveness and safety of photodynamic therapy versus laser resection in 31 patients with partial or complete tracheobronchial obstruction due to inoperable non-small cell lung cancer.

Fourteen patients received dihaematoporphyrin ether and argon dye laser photoradiation, and 17 patients received Nd-YAG laser resection. Endoluminal obstruction of $>75 \%$ was found in $77.4 \%$ of the patients. Among the symptoms, cough was more severe in the Nd-YAG group $(p=0.02)$.

Patients in both groups experienced symptomatic relief after treatment $(p=0.003)$. Patients in the photodynamic therapy (PDT) group showed a significantly longer time until treatment failure $(p=0.03)$ and longer median survival $(p=0.007)$. Bronchitis and photosensitization (both in the PDT group) were the most common adverse effects. There was one death, probably related to treatment, in the PDT group.

Photodynamic therapy and neodymium-yttrium aluminium garnet laser resection showed similar effectiveness and safety in the palliation of symptoms. The more prolonged survival in the photodynamic therapy group may have been due to differences in tumour stage between the groups. The degree of obstruction improved after treatment in both groups. In conclusion, photodynamic therapy is a valid method of palliation in partially or totally obstructing non-small cell lung carcinoma. Eur Respir J 1999; 14: 800-805.
\end{abstract}

Endoscopy and Laser Unit, Pulmonology Service, Ciutat Sanitària de Bellvitge, L' Hospitalet de Llobregat, Barcelona, Spain

Correspondence: J.P. Diaz-Jiménez, Endoscopy and Laser Unit, Pulmonology Service, Ciutat Sanitària de Bellvitge, Feixa Llarga s/n, E-08907 L'Hospitalet de Llobregat, Barcelona, Spain, Fax: 34932607745

Keywords: Advanced lung cancer haematoporphyrin derivative

laser palliation

laser photoresection

non-small cell lung cancer

photodynamic therapy

Received: October 61998

Accepted after revision May 261999

This study was supported by Lederle Pharmaceutical Company.
The majority of patients diagnosed with lung cancer have advanced disease, and at most $15-20 \%$ are surgical candidates at the time of diagnosis $[1,2]$. Most patients with inoperable tracheobronchial lesions will require palliative treatment with the primary goal of relieving an obstructed airway [3]. Neodymium-yttrium aluminium garnet (Nd-YAG) laser therapy is increasingly used in cases of nonresectable bronchogenic carcinoma [3, 4] and the results of different studies confirm the usefulness and safety of this method in relieving local symptoms and improving quality of life [5-7]. Photodynamic therapy (PDT) consists of the systemic administration of a photosensitizing agent, such as dihaematoporphyrin ether (DHE), followed by the delivery of light of a specific wavelength to the fluorescing tissue [8-11]. The photosensitizing agent is selectively retained in the cells and interstitial tissue of the tumour and together with argon dye laser photoradiation generates excited singlet oxygen, which produces a series of structural changes resulting in the destruction of the irradiated tissue $[10,12]$. Although a promising use of PDT appears to be in the treatment of early-stage lung cancer in carefully selected patients [1317] PDT has been shown also to have a role in the palliation of advanced inoperable obstructive bronchial tumours [4, 18-20). The aim of this study was to compare the effectiveness and safety of Nd-YAG laser resection and PDT using DHE as photosensitizer and argon dye laser radiation at $630 \mathrm{~nm}$ wavelength in the treatment of partially or totally obstruct ive tumours in patients with inoperable non-small cell carcinoma.

\section{Materials and methods}

\section{Study population}

Thirty-one patients with inoperable non-small cell lung cancer participated in a prospective controlled randomized study, and received either PDT or Nd-YAG laser resection and were followed for a period of 24 months. The groups were assigned by opening randomly ordered closed envelopes indicating the treatment modality. The study protocol was approved by the institutional Review Board and all patients or their legal representatives gave written informed consent.

Inclusion criteria were as follows: patients $>18$ yrs of age; nonpregnant, infertile or postmenopausal females. biopsy-proven or recurrent inoperable non-small cell lung cancer with totally or partially obstructive endobronchial lesions with or without extrabronchial tumour; clinical evidence of airway obstruction (dyspnoea, cough, haemoptysis); Karnofsky performance status $\geq 40 \%$; ability to tolerate bronchoscopic procedures and $\geq 4$ weeks from the last chemotherapy cycle and $\geq 3$ weeks from the last 
radiation dose. Patients were excluded if they had previously undergone PDT or Nd-YAG laser resection or if they had tracheal lesions that compromised both main bronchi, brain metastasis, bone pain due to skeletal metastasis, pneumonectomy, tumours eroding or invading great vessels, haematoporphyrin hypersensitivity, leukocyte count $<2 \times 10^{9}$ cells $\cdot \mathrm{L}^{-1}$ platelet count $<100 \times 10^{9}$ cells $\cdot \mathrm{L}^{-1}$ coagulation time $\geq 15 \mathrm{~min}$, renal failure (serum creatinine clearance $>3 \mathrm{mg} \cdot \mathrm{dL}^{-1}$ ) or liver dysfunction (two-fold increase in the upper limit of normal serum aminotransferases).

In all patients full staging was required. A detailed history and physical examination was recorded. Other investigations included electrocardiography (ECG) chest radiograph, chest computed tomography (CT) scans, preferably with contrast, routine laboratory tests, pulmonary function tests and assessment of the Karnofsky performance status. A baseline bronchoscopy was performed, recording the precise location and characteristics of the tumour, distance from the carina, approximate measurements using biopsy forceps and degree of airway obstruction (the smallest diameter of the tracheobronchial lumen was carefully measured) a biopsy of the tumour was also taken.

\section{Photodynamic therapy}

The PDT was based on the estimated size of the tumour, calculating the necessary power for the laser treatment. Since all tumours were $>0.5 \mathrm{~cm}$, a cylindrical diffusingtype fibre inserted into the tumour itself was used. Tumours were irradiated $(630-\mathrm{nm}$ light) via a flexible fibreoptic bronchoscope 40-50 h after intravenous injection of $2 \mathrm{mg} \cdot \mathrm{kg}^{-1}$ DHE (Photofrin ${ }^{\mathbb{R}}$; Lederle, Vancouver, Canada). Two days after the treatment, a repeat bronchoscopy was performed in order to clean detritus. A second argon dye laser irradiation was performed if parts of the tumour failed to show signs of necrosis 96-120 h after irradiation. When symptoms recurred within 30 days after treatment and a further bronchoscopy revealed local recurrence, patients could receive a second session of PDT that consisted of the same dose of DHE followed by laser photoradiation, as initially described. Patients could receive a maximum of three doses of DHE at 1-month intervals and up to six laser photoradiations, with a maximum of two photoradiations per session. If toxic effects occurred treatment was stopped until these resolved.

\section{Neodymium-yttrium aluminium garnet laser resection}

Bronchoscopy was performed using a rigid bronchoscope and standard techniques under general anaesthesia. Nd-YAG resection was performed using $15-80-$ W pulses and a pulse duration of $0.5-1.5 \mathrm{~s}$. Coagulated parts of the tumour were extracted immediately. Bronchoscopy was repeated 2-4 days later to assess the results of treatment and to remove detritus. Bronchoscopy and Nd-YAG laser sessions were repeated every 2-4 days until it was considered that further application would not give additional benefits. If symptoms worsened or recurred and tumour regrowth was confirmed, further Nd-YAG laser treatment was indicated.
Control bronchoscopy after either PDT or Nd-YAG laser resection was performed 1 week after PDT, every month for 3 months and at 6 and 12 months (and at 18 months, if possible) thereafter. A complete response (CR) was defined as the absence of tumour on bronchoscopy and biopsy, or the absence of tumour on bronchoscopy and evidence of malignancy on biopsy specimens (CR1 and CR2, respectively). A partial response was defined as a reduction in either the tumour length or the product of length and width, or as an improvement in the smallest diameter of the tracheobronchial lumen or the visualization of a new lumen in cases of complete obstruction of the airway. Progression of disease was defined as an increase of $\geq 25 \%$ in the tumour length or in the product of length and width, or a reduction of $\geq 50 \%$ in the smallest tracheobronchial diameter or recurrence of the tumour after a CR. Stable disease was defined by minor changes in tumour size and/or the smallest tracheobronchial diameter.

\section{Evaluation of effectiveness}

Treatment was evaluated in two main ways: the length of time from treatment to local recurrence (i.e. tumour recurrence at the treated site) and change in symptoms (improvement or worsening of cough, dyspnoea, haemoptysis, etc.). Secondary measurements included: objective measurement of the response of the tumour (size reduction) by means of bronchoscopy and CT scan, changes in pulmonary function test results, and length of time from treatment to failure due to progression of the disease, toxicity due to the therapy, death or any other reason, such as the patient's request to withdraw from the study. Quality of life was followed using Karnofsky performance status before treatment and at 1 week and 1, 2, 3, 6, 12 and 18 months thereafter. Patients were withdrawn from the study in case of: 1) no evidence of palliation of symptoms or objective improvement (no variation in size) after two PDT or four Nd-YAG laser sessions; 2) unacceptable toxicity; or 3) on the patient's request.

\section{Evaluation of safety}

Any adverse reaction unrelated to, or potentially associated with, the use of DHE was care fully recorded, along with the date of onset, intensity, treatment and outcome. Laboratory results were followed carefully. If any value was abnormal after the treatment, a causal relationship was considered and the safety of treatment continuation evaluated.

\section{Statistical analysis}

Data were analysed using the SAS (Cari, NC, USA) and SPSS (Chicago, IL, USA) statistical software packages for Windows and expressed as percentages or mean \pm SD. Because of sample size, nonparametric tests were used: MannWhitney U-test, Kruskal-Wallis test and the Wilcoxon test to compare means. Proportions were compared using the Chi-squared test (and Yate's correction when needed) Fisher's exact test was used when the expected survival rate was estimated via the Kaplan-Meier method. Differences in the probability of survival were assessed using the log-rank test. Statistical significance was set at $p<0.05$. 


\section{Results}

Of the 31 patients included in the study, 14 were randomly assigned to the PDT group and 17 to the Nd-YAG laser resection group. Patients included in this study were not considered surgical candidates because of poor pulmonary status and/or adverse staging.

All patients were male with a mean \pm SD age of $65 \pm 7$ yrs. The distribution of patients according to the presence of contralateral pulmonary metastases and dyspnoea on minimal effort was similar in both groups. For the analysis of the results only the therapeutic modality was considered (PDT versus Nd-YAG).

The mean age of the patients was $67 \mathrm{yrs}$ in the PDT group and 64 yrs in the Nd-YAG laser group. Other baseline characteristics, such as Karnofsky performance status and results of laboratory and pulmonary function tests, were also similar. Cough, however, was more common in the Nd-YAG laser resection group $(\mathrm{p}=0.02)$.

Staging of the patients is shown in table 1. By chance, the PDT group contained fewer patients with advanced disease.

The tumour affected the right main bronchus in 12 cases, the left main bronchus in eight, the left superior bronchus in five, the right inferior bronchus in three, the intermediate bronchus in two, and the left inferior bronchus in one. The mean distance from the tracheal carina was $4.4 \mathrm{~cm}$ in the PDT group and $2.2 \mathrm{~cm}$ in the Nd-YAG laser resection group $(\mathrm{p}=0.1)$. The distribution of tumour length and degree of airflow obstruction was similar in both groups. The tumour length ranged $1.0-1.9 \mathrm{~cm}$ in five patients, $2.0-2.9$ in five and $3.0-3.9 \mathrm{~cm}$ in five. In nine patients in the PDT group and seven in the Nd-YAG laser resection group, the length of the tumour could not be determined. Lesions caused $>75 \%$ bronchial obstruction in 24 patients and $<75 \%$ in the remaining seven; the distribution was similar in both groups. Squamous cell carcinoma was found in $25(80.6 \%)$ of the 31 patients (table 1$)$.

Five patients had received previous treatment: three patients in the PDT group received external radiotherapy; one patient in the Nd-YAG laser group had received combined chemotherapy and external radiation; and the remaining one, also in the Nd-YAG laser group, had un-

Table 1. - Pretreatment characteristics of 31 patients with inoperable non-small cell lung cancer

\begin{tabular}{lcc}
\hline & \multicolumn{2}{c}{ Group } \\
\cline { 2 - 3 } Data & PDT & $\begin{array}{c}\text { Nd-YAG laser } \\
\text { resection }\end{array}$ \\
\hline Patients n & 14 & 17 \\
Staging & 3 & 1 \\
I & 1 & 0 \\
II & 2 & 4 \\
IIIA & 3 & 7 \\
IIIB & 3 & 4 \\
IV & 2 & 1 \\
Recurrent tumour & & \\
Histological type & 13 & 12 \\
Squamous cell carcinoma & 1 & 2 \\
Adenocarcinoma & 0 & 3 \\
Undifferentiated carcinoma & 0 & \\
\hline
\end{tabular}

PDT: photodynamic therapy; Nd-YAG: neodymium-yttrium aluminium garnet. dergone exploratory thoracotomy before the study. The periods from the last treatment session were 11, 41, 140 and 114 weeks for the four patients who had received radiation therapy, and 40 weeks from the last chemotherapy session in the patient who had received combined radiation and chemotherapy. The patient who had undergone thoracotomy was found to have advanced disease at the time of the surgery, and no tumour resection was performed.

Patients were treated according to the described protocol. Five patients in the PDT group required a second dose of DHE $1(\mathrm{n}=2), 3(\mathrm{n}=1)$, and 4 months $(\mathrm{n}=1)$ and $1 \mathrm{yr}$ $(\mathrm{n}=1)$ following the first treatment. Only one patient in the $\mathrm{Nd}$-YAG laser therapy group required a second laser photoresection, 45 days after the first treatment.

The median time elapsed until treatment failure was 50 days in the PDT group versus 38 days in the Nd-YAG laser resection group $(\mathrm{p}=0.03)$. Causes of failure in the PDT group included failure of the treatment to work or patient request in three cases, objective tumour progression in five, systemic progression of the disease in one, worsening of symptoms in two, withdrawal to receive chemotherapy in one, adverse effects related to the treatment in one, and death in one. In the Nd-YAG laser resection group, causes of failure were: failure of the treatment to work or patient request in six cases, objective tumour progression in four, systemic progression in one, worsening of symptoms in three, and death in three.

The response to the treatment was similar in both groups. At the 1-week follow-up examination, the response rate was $43 \%$ in the PDT group versus $53 \%$ in the NdYAG laser resection group; the corresponding figures at 1 month were $38.5 \%$ versus $23.5 \%$, $(\mathrm{p}=\mathrm{NS})$ (table 2$)$. Amelioration of symptoms was similar in both groups. Dyspnoea, haemoptysis, cough, and sputum production improved within 1 week after treatment; between 1 week and 1 month post-treatment, dyspnoea, haemoptysis and sputum production showed greater improvement than did cough. Airway obstruction improved from $79 \%$ to $62 \%$ in the 27 patients who completed the follow-up examinations $(\mathrm{p}=0.03)$.

A total of $26(84 \%)$ patients had at least one adverse effect, bronchitis being the most common (four cases in the

Table 2. - Tumour size at first follow-up bronchoscopy

\begin{tabular}{|c|c|c|c|c|}
\hline \multirow[b]{3}{*}{ Type of response } & \multicolumn{4}{|c|}{ Group } \\
\hline & \multicolumn{2}{|c|}{ PDT } & \multicolumn{2}{|c|}{$\begin{array}{l}\text { Nd-YAG laser } \\
\text { resection }\end{array}$} \\
\hline & 1 week & 1 month & 1 week & 1 month \\
\hline Patients $\mathrm{n}$ & \multicolumn{2}{|c|}{14} & \multicolumn{2}{|c|}{17} \\
\hline Partial response & 6 & 3 & 8 & 4 \\
\hline Stable disease & 6 & 2 & 4 & \\
\hline Progressive disease & 1 & 3 & & 5 \\
\hline Complete response $1^{*}$ & & 1 & & \\
\hline Complete response $2^{+}$ & & 1 & 1 & \\
\hline Unclassified $^{\#^{1}}$ & & 3 & 4 & 8 \\
\hline Death & 1 & & & \\
\hline
\end{tabular}

*:No tumour on bronchoscopy and biopsy; ${ }^{+}$:No tumour on bronchoscopy, evidence of malignancy on biopsy specimens; ${ }^{*}$ : Unable to tolerate bronchoscopy or failure to attend follow-up appointments. PDT: photodynamic therapy; Nd-YAG: neodymium-yttrium aluminium garnet. 
PDT group, one in the Nd-YAG laser group), followed by photo sensitization in four patients in the PDT group and dyspnoea in three patients. Sixteen patients had experienced two adverse effects, cough and photosensitization being the most frequent combination. Five patients, all in the Nd-YAG laser resection group, experienced no adverse events. One treatment was discontinued due to photosensitization.

Survival was significantly longer in the PDT group than in the Nd-YAG laser resection group (265 versus 95 days, $\mathrm{p}=0.007$ ) (fig. 1)

Five patients died within 2 months of the last day of treatment, one in the PDT group (probably related to treatment) and four in the Nd-YAG laser group. By the end of the study, 23 (74.2\%) of the 31 patients had died, 19 due to progression of malignancy (seven in the PDT group, 12 in the Nd-YAG laser group), one probably related to treatment (PDT group) and three for unknown reasons. Two patients (one in each group) died from massive haemoptysis and presumed progression of the disease, although no post mortem examination was performed. Eight patients, four in each group, were still alive at the end of the study.

\section{Discussion}

This study was designed to evaluate the effectiveness and safety of PDT using an argon dye laser and DHE versus Nd-YAG laser resection in the management of patients with inoperable non-small cell lung cancer. Patients with small cell lung cancer were not included because of the high response rate of this histological type to chemotherapy. In addition, small cell carcinoma rarely presents as an obstructive mass. Involvement of the two main bronchi was another exclusion criterion, since a significant and important inflammatory reaction occurs immediately after PDT, with marked oedema, which may further reduce endoluminal obstruction and place the patient at risk of respiratory insufficiency.

It was found that the length of time from treatment to recurrence of symptoms or treatment failure and the survival period were significantly longer for patients in the PDT group. These findings are difficult to interpret in the

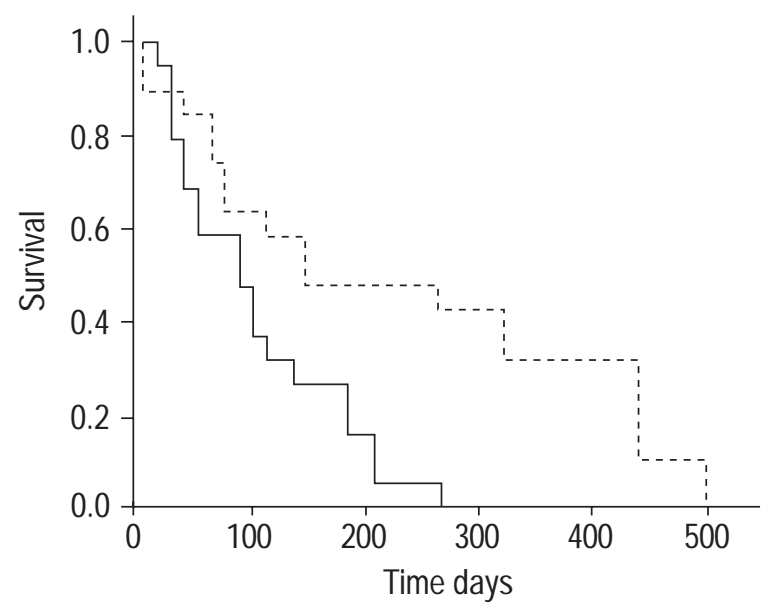

Fig. 1. - Probability of survival of patients with inoperable non-small cell lung cancer assigned to photodynamic therapy (- - ) and Nd-YAG laser resection (- $)$. light of the different distribution of staging between the groups, but one of the reasons could be more selective destruction of neoplastic tissue by the photochemical reaction than achieved by means of Nd-YAG laser therapy [4]. The degree of obstruction improved significantly after treatment in both groups. More patients in the PDT group required a second treatment.

The selectivity of haematoporphyryn derivatives for neoplastic tissues is a controversial point. Pharmacokinetic studies in humans [21] have suggested that there is no difference in the drug uptake of tumourous and normal tissues, and that selectivity, if any, is maximal within 1 or $2 \mathrm{~h}$ after drug injection rather than $24 \mathrm{~h}$ later (which is the usual timing of photoradiation). The fact that tumour recurrence has been reported to occurr later in the PDTtreated patients versus those treated with Nd-YAG laser $[4,8,18]$ favours the concept that some degree of selectivity indeed exists. However, this point remains to be further investigated.

It is well known that haematoporphyrin derivatives produce skin photosensitization that can persist for 4-6 weeks after treatment. Precautions should be taken to avoid exposure to the sun or other sources of potent light. Patients are also advised to cover their skin and wear sunglasses for 30 days after injection of DHE. After this period, a small area of skin can be exposed to the sun to assess the presence and degree of residual photosensitization.

Five patients died within two months of treatment. The death in the PDT group was considered to be probably related to treatment. This patient had undergone two photoradiation sessions after the DHE injection, and the followup bronchoscopic examination could not be performed due to severe dyspnoea. The post mortem examination showed an exophytic mass which had apparently increased in size since the pre-treatment assessment. The other four deaths occurred in the Nd-YAG laser resection group and were due to progression of the disease. Two further patients died because of massive haemoptysis, one in each group; and this was also considered to be due to progression of the disease.

PDT has been used in research on the treatment of lung cancer since 1980 [15], and is used as a treatment has progressively increased. The best results are obtained in early-stage lung carcinoma (limited to the mucosa). Previous reports have included a total of 178 lesions treated with curative purpose [22-26]. The range of CRs was 64$100 \%$. In the recent study published by CORTESE et al. [14], 23 early lesions were treated. After PDT, the followup period was 24-116 months. A CR was identified in $71 \%$ of the patients, but only $52 \%$ had a response that lasted $>12$ months, and ultimately $43 \%$ of the patients were spared surgical resection.

Clinical studies in advanced inoperable obstructive bronchial tumours have shown that significant palliation can be achieved. LOCICERO et al. [20] reported on 10 patients with late-stage advanced disease, finding a significant reduction in the obstruction from $86 \pm 2 \%$ to $57 \pm 3 \%$. All patients demonstrated a decrease in symptoms, particularly cough and dyspnoea. Pulmonary function tests preand post-treatment did not show significant changes. This study reported a similar survival rate to that of the present PDT group. In the group of LoCICERO et al. [20], 40\% of patients had previously been treated with external radiation, versus $21 \%$ in the present PDT group. The interval 
between the last radiation session and the PDT treatment was not specified in the study of LoCiCERO et al. [20]. SuTEDJA et al. [19] reported a partial response rate of $73 \%$ in stage III patients, with improvement in dyspnoea in $58 \%$ of the partial responders. CR, however, was found only in Stage I disease. Pulmonary function test results did not improve in this study either.

Four CRs (three in two patients in the PDT group and one in the Nd-YAG laser group) were found, but they were not sustained for a prolonged period. In the patient in the $\mathrm{Nd}$-YAG laser resection group, a CR at 1 week was followed by progressive disease at the next examination at 1 month. Two patients from the PDT group showed stable disease at 1 week and CR at 1 month, but these responses were again not sustained, with progressive disease demonstrable at two months. The delay in the response was probably due to the characteristics of the photodynamic reaction, which slowly makes the injected photosensitizer cytotoxic. Similar findings have been reported by others [8].

McCaughan and Williams [18] reported on 175 nonsurgical lung cancer patients treated with PDT, finding that the median survival was 7 months, and that the survival rate for patients with advanced disease (IIIA, IIIB and IV) was significantly lower in those with poor functional status (Karnofsky score $<50$ ).

A relationship was found between response and tumour size, a finding supported by other authors [23]. The three patients with CRs had small tumours $(1 \times 0.5 \times 0.3 \mathrm{~cm}$. $2 \times 0.2 \times 0.2 \mathrm{~cm}$ and $1 \times 1 \times 0.2 \mathrm{~cm})$. The overall response rate was $43 \%$ at 1 week and $38.5 \%$ at 1 month in the PDT group, and $53 \%$ and $23.5 \%$, respectively, in the Nd-YAG laser resection group. It should be noted that a better immediate response was obtained in the Nd-YAG laser resection group as opposed to a more favourable delayed response in the PDT group due to the different mechanisms of action of the two procedures. However, the difference was not statistically significant. This finding was also noted by McCAughan et al. and WiLliams [18] in a report comparing PDT therapy versus Nd-YAG laser therapy $[8,27]$, in which reopening of the airway was more significant at the end of Nd-YAG laser therapy than in those treated by using PDT, and reobstruction after NdYAG laser therapy occurred more rapidly than in the PDT-treated group, when compared 2 and 4 weeks after treatment. With regard to airway patency, an immediate improvement from 79 to $56 \%$ obstruction in the Nd-YAG laser resection group versus 79 to $71 \%$ obstruction in the PDT group was noted in the present study.

In conclusion, PDT and conventional Nd-YAG laser therapy appeared to be equally effective and safe in relieving airway obstruction in patients with inoperable nonsmall cell lung cancer. An advantage of PDT was the longer time to treatment failure and the lower survival; a disadvantage was the higher incidence of adverse effects, particularly photosensitization. Nd-YAG laser resection, however, was found to achieve a better rate of immediate reopening, and it seemed to be more suitable when the tracheobronchial obstruction required rapid relief. In this respect, none of the patients in the present study were in a situation in which immediate reopening of the airway was required. Based on the known mechanism of action of both treatments and the authors' experience, the use of PDT should be discouraged in incipient respiratory insufficiency due to tumoural airway obstruction. Other methods, NdYAG laser therapy among them, are indicated in such circumstances.

The statistical significance of these findings needs to be analysed in the light of two factors: first, the small number of patients involved in the present study, and, secondly, the random distribution allocated less advanced disease to the photodynamic therapy group. The prolonged survival found in this group in part, may be, due to this unequal distribution. However, the improvement in airway obstruction and palliation of symptoms were significant in both groups. Photodynamic therapy has been shown to be a valid means of palliation, comparable in effectiveness to the neodymium-yttrium aluminium garnet laser. Its advantage over other available palliation therapies remains to be proven.

\section{References}

1. Fry WA, Menck HR, Winchester DP. The National Cancer Data Base Report on Lung Cancer. Cancer 1996; 77 : 1947-1955.

2. Whitehouse JMA. Management of lung cancer. Report of working group. Current Clinical Practices 1994; 1.

3. Carrol M, Morgan S, Yarnold JA, Hill JM, Wright NM. Prospective evaluation of a watch policy in patients with inoperable non-small cell cancer. Eur J Cancer Clinic Oncol 1986; 22: 1352-1356.

4. Moghissi K, Dixon K, Hudson E, Stringer M, Brown S. Endoscopic laser therapy in malignant tracheobronchial obstruction using sequential Nd-YAG laser and photodynamic therapy. Thorax 1997; 52: 281-283.

5. Diaz-Jimenez JP, Martinez-Ballarin JI, Farrero-Muñoz E, Kovitz KL, Castro-Serrano MJ. Video Endoscopy for laser photoresection in tracheobronchial pathology: some considerations after 9 years experience with 2105 treatments. Diagnos Therap Endosc 1995; 2: 79-87.

6. Cavallieri S, Foccoli P, Toninelli C, et al. Nd-YAG laser therapy in lung cancer: an 11-year experience with 2,253 applications in 1,585 patients. $J$ Bronchol 1994; 1: 105111.

7. Dumon JF, Reboud E, Garbe L, Aucomte F, Meris B. Treatment of tracheobronchial lesions by laser photoresection. Chest 1982; 81: 278-284.

8. Dougherty TJ, Gomer CJ, Henderson BW, et al. Photodynamic therapy. J Natl Cancer Inst 1998; 90: 889-905.

9. Edell ES, Cortese DA. Photodynamic Therapy. Clin Chest Med 1995; 16: 455-463.

10. Henderson BW, Bellnier DA. Tissue localisation of photosensitizers and the mechanism of photodynamic tissue destruction. Ciba Found Symp 1989; 146: 112-125.

11. Jori G. Tumor photosensitizers: approaches to enhance the selectivity and efficiency of photodynamic therapy. $J$ Photochem Photobiol B 1996; 36: 87-93.

12. Henderson BW, Fingar VH. Oxygen limitation of direct tumor cell kill during photodynamic treatment of a murine tumor model. Photochem Photobiol 1989; 49: 299-304.

13. Hayata $\mathrm{Y}, \mathrm{Kato} \mathrm{H}$, Konaka $\mathrm{C}$ et al. Photoradiation therapy with hematoporphyrin derivative in early stage I lung cancer. Chest 1984; 86: 169-177.

14. Cortese DA, Edell ES, Kinsey JH. Photodynamic Therapy for early stage squamous cell carcinoma of the lung. Mayo Clin Proc 1997; 72: 595-602.

15. Cortese DA, Kinsey JH, Woolner LB, et al. Clinical application of a new endoscopic technique for detection of in situ bronchial carcinoma. Mayo Clin Proc 1979; 54: 635-641. 
16. Vermilen P, Berghans T, Roufosse C, et al. Diagnosis and treatment of early-stage bronchial cancer: current status. Rev Med Brux 1997; 18: 347-352.

17. Hayata Y, Kato H Konakla C, et al. Photoradiation therapy in early stage cancer of the lung, esophagus and stomach. In: Cubbedu AA, ed. Porphyrins in Tumor Phototherapy: New York Plenum, 1984: 405-412.

18. McCaughan JS Jr., Williams TE. Photodynamic therapy for endobronchial malignant disease: a prospective fourteen-year study. J Thorac Cardiovasc Surg 1997; 114: 940-946. discussion 946-947.

19. Sutedja T, Baas P, Stewart F, van Zandwijk N. A pilot study of photodynamic therapy in patients with inoperable non-small cell lung cancer. Europ J Cancer 1992; 28A: $1370-1373$.

20. LoCicero J, Metzdorff M, Almgren C. Photodynamic therapy in the palliation of late stage obstructing nonsmall cell lung cancer. Chest 1990; 98: 97-100.

21. Braichotte DR, Wagnieres GA, Bays R, Monnier P, van den Bergh HE. Clinical pharmacokinetic studies of photofrin by fluorescence spectroscopy in the oral cavity, the esophagus and the bronchi. Cancer 1996; 75: 27682778 .
22. Edell ES, Cortese DA. Photodynamic therapy in the management of early superficial squamous cell carcinoma as an alternative to surgical resection. Chest 1992; 102: 1319-1322.

23. Furuse K, Fukuoka M, Kato $\mathrm{H}$, et al. A prospective phase II study on photodynamic therapy with Photofrin II for centrally located early-stage lung cancer. J Clin Oncol 1993; 10: 1844-1845.

24. Okunaka T, Kato H, Konaka C, et al. Photodynamic therapy for multiple primary, bronchogenic carcinoma. Cancer 1991; 68: 253-258.

25. Imamura S, Kusunoki Y, Takifuji N, et al. Photodynamic therapy and/or external beam radiation therapy for roentgenologically occult lung cancer. Cancer 1994; 73: 16081614.

26. Sutedja G. Response and pattern of failure after photodynamic therapy for intraluminal stage I lung cancer. $J$ Bronchol 1994; 1: 295-298.

27. McCaughan JSJ. Photodynamic therapy versus ND-YAG laser treatment of endobronchial or esophageal malignancies. In: Spinelli P, Del Fante M, Marchesini R, Del Fante M, eds. Photodynamic Therapy and Biomedical Lasers. New York: Elsevier, 1992; 23-26. 